\title{
A Gaussian Distribution for Refined DT Invariants and 3D Partitions
}

\section{Journal Article}

Author(s):

Morrison, Andrew

Publication date:

2014-11

Permanent link:

https://doi.org/10.3929/ethz-b-000088661

Rights / license:

In Copyright - Non-Commercial Use Permitted

Originally published in:

Communications in Mathematical Physics 331(3), https://doi.org/10.1007/s00220-014-2051-8 


\title{
A Gaussian Distribution for Refined DT Invariants and 3D Partitions
}

\section{Andrew Morrison}

ETH Zurich, Zurich, Switzerland. E-mail: andrewmo@math.ethz.ch

Received: 22 June 2013 / Accepted: 1 November 2013

Published online: 26 April 2014 - (C) Springer-Verlag Berlin Heidelberg 2014

\begin{abstract}
We show that the refined Donaldson-Thomas invariants of $\mathbb{C}^{3}$, suitably normalized, have a Gaussian distribution as limit law. Combinatorially, these numbers are given by weighted counts of 3D partitions. Our technique is to use the Hardy-Littlewood circle method to analyze the bivariate asymptotics of a $q$-deformation of MacMahon's function. The proof is based on that of E.M. Wright, who explored the single variable case.
\end{abstract}

\section{Introduction}

In [6] physicists suggested that the string theory associated to a Calabi-Yau threefold $X$ should produce an algebra of BPS states:

$$
\mathcal{H}_{B P S}=\bigoplus_{\gamma \in \Gamma} \mathcal{H}_{\gamma}
$$

where here the (charge) lattice $\Gamma$ is identified with the even cohomology of $X, \Gamma=$ $\bigoplus_{i=0}^{3} H^{2 \mathrm{i}}(X, \mathbb{Z})$. Moreover, each individual vector space $\mathcal{H}_{\gamma}$ should have an additional $\mathbb{Z}$-grading coming from a symmetry in the little group Spin(3) [5].

Mathematically, we consider the cohomological Hall algebra [10] as giving the algebra of BPS states on $X=\mathbb{C}^{3}$. In this case $\Gamma=H^{6}(X, \mathbb{Z})=\mathbb{Z}$ and the $\gamma=n$th piece is given by the critical cohomology of $\operatorname{Hilb}^{n}\left(\mathbb{C}^{3}\right) .{ }^{1}$ Moreover, each of these vector spaces has a cohomological $\mathbb{Z}$-grading. The Betti numbers of these graded pieces are know as refined DT invariants. These numbers are dependent on the singularities of $\operatorname{Hilb}^{n}\left(\mathbb{C}^{3}\right)$.

However, on a recent visit to EPFL, T. Hausel shared with me the output of a computer experiment. He conjectured that the refined DT invariants, suitably normalized, would have a Gaussian distribution as limit law, i.e., for large $n$ plotting the Betti numbers

\footnotetext{
${ }^{1}$ In addition, we add a single D6-brane filing the $\mathbb{C}^{3}$ or mathematically a framing.
} 
$\begin{array}{llll}0 & 0 & 0 & 0\end{array}$

$\begin{array}{llllll}2 & 2 & 1 & 0 & 0 & \cdots\end{array}$

$\begin{array}{llllll}3 & 3 & 2 & 0 & 0 & \cdots\end{array}$

$\begin{array}{lllllll}4 & 3 & 2 & 1 & 0 & \cdots\end{array}$

$\begin{array}{llllllll}5 & 3 & 2 & 1 & 1 & 0 & \cdots\end{array}$

Fig. 1. Integers

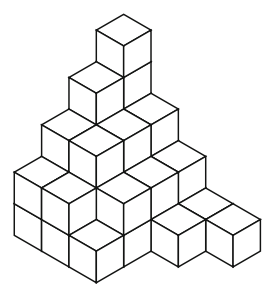

Fig. 2. Boxes

against cohomological degree should give the bell curve of a Gaussian distribution (cf. [14]). The goal of this paper is to prove that conjecture.

In fact this proposal is entirely combinatorial. The Hilbert-Poincare series for the cohomological Hall algebra, computed in [2], equals $M_{3}\left(t, q^{1 / 2}\right)$, where $t$ gives the $\Gamma$-grading, $q$ gives the cohomological grading, and

$$
M_{\delta}(t, q)=\prod_{m \geq 1} \prod_{k=0}^{m-1} \frac{1}{1-q^{\delta+2 k+1-m} t^{m}} .
$$

Expanding this series gives an explicit formula [12] for the $t^{n}$ coefficient

$$
\sum_{\pi \vdash n} q^{\delta w_{0}(\pi)+w_{+}(\pi)-w_{-}(\pi)}
$$

where the sum is over all plane partitions of $n$, which we now explain.

A plane partition is given by a two dimensional array of positive integers in the first quadrant of $\mathbb{Z}^{2}$ that are weakly decreasing in both the $x, y$ directions. In this way plane partitions are a generalization of ordinary (line) partitions [1]. The analogue of the Young diagram in this situation is a stack of three dimensional boxes in $\mathbb{Z}_{\geq 0}^{3}$. Such a collection gives a plane partition if and only if the stack is stable under the pull of gravity along the $(1,1,1)$ axis. For example, let $\pi$ be the plane partition given alternatively as Figs. 1 and 2 .

Here the total sum of the integers/boxes is $|\pi|=35$ and we say that the partition has size 35. The statistics appearing in the above formula for refined DT invariants are

$$
w_{+}(\pi)=\sum_{i<j} \pi_{i, j}, \quad w_{-}(\pi)=\sum_{i>j} \pi_{i, j}, \quad w_{0}(\pi)=\sum_{i} \pi_{i, i} .
$$

Considering the set $\mathcal{P}_{n}$ of plane partitions of size $n$ as a sample space with uniform measure, we define three random variables

$$
X_{n}^{+}, X_{n}^{-}, X_{n}^{0}: \mathcal{P}_{n} \rightarrow \mathbb{R}
$$

given by $w_{+} / n^{2 / 3}, w_{-} / n^{2 / 3}$, and $w_{0} / n^{2 / 3}$. Our main result is the following: 
Theorem 1.1. The distribution of the random variable

$$
\delta \cdot X_{n}^{0}+X_{n}^{+}-X_{n}^{-}
$$

for large $n$ has the Gaussian distribution as limit law with

$$
\mu=\delta \zeta(2) /(2 \zeta(3))^{2 / 3} \text { and } \sigma^{2}=1 /(2 \zeta(3))^{1 / 3}
$$

\section{Setup}

First we split the problem into two parts, one of which has already been solved. Straight away we see that the covariance of $X_{n}^{0}$ and $X_{n}^{+}-X_{n}^{-}$is zero due to symmetry

$$
\begin{aligned}
\mathbb{E}\left(\left(X_{n}^{0}-\mu_{X_{n}^{0}}\right)\left(X_{n}^{+}-X_{n}^{-}-\mu_{X_{n}^{+}-X_{n}^{-}}\right)\right) & =\mathbb{E}\left(\left(X_{n}^{0}-\mu_{X_{n}^{0}}\right)\left(X_{n}^{+}-X_{n}^{-}\right)\right) \\
& =\mathbb{E}\left(X_{n}^{0}\left(X_{n}^{+}-X_{n}^{-}\right)\right)-\mathbb{E}\left(\mu_{X_{n}^{0}}\left(X_{n}^{+}-X_{n}^{-}\right)\right) \\
& =\mathbb{E}\left(X_{n}^{0} X_{n}^{+}-X_{n}^{0} X_{n}^{-}\right)-\mu_{X_{n}^{0}}\left(\mathbb{E}\left(X_{n}^{+}-X_{n}^{-}\right)\right) \\
& =0-0 .
\end{aligned}
$$

The following is a result of E.P. Kamenov and L.R. Mutafchiev:

Theorem 2.1 [9]. Let $a=\zeta(2) /(2 \zeta(3))^{2 / 3}$ and $b=\sqrt{1 / 3} /(2 \zeta(3))^{1 / 3}$. Then as $n \rightarrow \infty$ we have

$$
X_{n}^{0} \sim \mathcal{N}\left(a, b n^{-\frac{1}{3}} \ln ^{\frac{1}{2}} n\right) .
$$

Now $X_{n}^{0}$ converges in distribution to the constant $a$. Therefore the joint distributions of $\left(X_{n}^{+}-X_{n}^{-}, X_{n}^{0}\right)$ and $\left(X_{n}^{+}-X_{n}^{-}, a\right)$ converge to the same limit. In particular, the limiting distribution of $\delta \cdot X_{n}^{0}+X_{n}^{+}-X_{n}^{-}$will equal that of $\delta \cdot a+X_{n}^{+}-X_{n}^{-}$. Theorem 1.1 will now follow from the result just mentioned together with:

Theorem 2.2. Let $c=1 /(2 \zeta(3))^{1 / 3}$. Then as $n \rightarrow \infty$ we have

$$
X_{n}^{+}-X_{n}^{-} \sim \mathcal{N}(0, c) .
$$

To prove this result we use the method of moments. That is we show that the limiting distribution has the same moments as a Gaussian random variable with variance $1 /(2 \zeta(3))^{1 / 3}$. Specifically we will show that in the limit

$$
\mathbb{E}\left(\left(X_{n}^{+}-X_{n}^{-}\right)^{k}\right)=\left\{\begin{array}{cl}
0 & \text { if } k \text { is odd } \\
(k-1) ! !(2 \zeta(3))^{-k / 6} & \text { if } k \text { is even }
\end{array}\right.
$$

Consider the generating series

$$
M_{0}(t, q)=\prod_{m \geq 0} \prod_{k=0}^{m-1} \frac{1}{1-q^{2 k+1-m} t^{m}}=\sum_{\pi} q^{w_{+}(\pi)-w_{-}(\pi)} t^{|\pi|}
$$

and let $p_{n}(q)$ be the coefficient of $t^{n}$ then we have

$$
\mathbb{E}\left(\left(X_{n}^{+}-X_{n}^{-}\right)^{k}\right)=n^{-2 k / 3} \frac{\left.\partial^{k} p_{n}(q)\right|_{q=1}}{\left.p_{n}(q)\right|_{q=1}}
$$


where $\partial=q \frac{d}{d q}$. Notice by symmetry this already implies that all the odd moments vanish. The method of proof given in the next section follows the proof of E.Wright [15] who provided an asymptotic formula ${ }^{2}$ for the number of plane partitions of $n$

$$
\left.p_{n}(q)\right|_{q=1} \sim \frac{\zeta(3)^{7 / 36}}{2^{11 / 36} \sqrt{3 \pi} n^{25 / 36}} e^{3\left(\frac{\zeta(3)}{4}\right)^{1 / 3}\left(n^{4 / 3}\right)^{1 / 2}+\zeta^{\prime}(-1)} .
$$

Wright's proof in turn generalized the pioneering work of Hardy and Ramanujan [7] who first applied the Hardy-Littlewood circle method to get an asymptotic formula for the number of ordinary partitions. Using this method in the next section we will show that

$$
\left.\partial^{k} p_{n}(q)\right|_{q=1} \sim n^{2 k / 3} \cdot(k-1) ! !(2 \zeta(3))^{-k / 6} \cdot \frac{\zeta(3)^{7 / 36}}{2^{11 / 36} \sqrt{3 \pi} n^{25 / 36}} e^{3\left(\frac{\zeta(3)}{4}\right)^{1 / 3}\left(n^{4 / 3}\right)^{1 / 2}+\zeta^{\prime}(-1)}
$$

when $k$ is even. This gives the correct moments and shows that $X_{n}^{+}-X_{n}^{-}$has a Gaussian limit law as promised.

\section{Proof}

As explained in the previous section we are going to use the Hardy-Littlewood circle method to estimate the coefficients in the generating series

$$
M_{k}(t):=\left.\partial^{k} M_{0}(t, q)\right|_{q=1} .
$$

Given any function $A(t)=\sum_{n>0} a_{n} t^{n}$ analytic on the interior of the unit disk we can compute the $n$th coefficient in its MacLaurin series using the Cauchy formula

$$
a_{n}=\frac{1}{2 \pi i} \int_{C_{N}} t^{-n-1} A(t) d t
$$

where $C_{N}$ is the circle or radius $e^{-1 / N}$. The idea of the circle method is that by understanding the singularities of $A$ on the unit circle one can approximate this integral by an integral over a small subarc of the circle when $N, n \gg 1$.

For example when $A(t)=M(t)=\left.M(t, q)\right|_{q=1}$ is MacMahon's function then letting $t=e^{z}$ Wright defines the major $\operatorname{arc} C_{N}^{\prime}$ to be the points such that $\operatorname{im}(z)<1 / N$ and the minor $\operatorname{arc} C_{N}^{\prime \prime}$ to be the remaining points on the circle $C_{N}$. In some sense MacMahon's function is most singular at $z=0$ and so the integral over $C_{N}$ is well approximated by that over the small arc $C_{N}^{\prime}$. The following two Lemmas ${ }^{3}$ make this precise and will be very useful to us later:

Lemma 3.1 (E.M. Wright [15] Lemma I). There exists constants $N_{0}, K$ so that for all $N>N_{0}$ and $t=e^{z} \in C_{N}^{\prime}$ along the major arc we have

$$
\left|M(t)-e^{-\zeta^{\prime}(1)} z^{1 / 12} e^{\zeta(3) / z^{2}}\right|<K N^{-1 / 12-2} e^{\zeta(3) N^{2}} .
$$

\footnotetext{
2 Typo warning: Wrights formula on page 179 is missing factor of $\sqrt{3}$ found at the end of his proof on page 189.

${ }^{3}$ Wright's first Lemma is more refined than this. We merely extract his leading order approximation suitable for our purposes.
} 
Lemma 3.2 (E.M. Wright [15] Lemma II). Given any $\epsilon>0$ there exists an $N_{0}$ such that for all $N>N_{0}$ and $t \in C_{N}^{\prime \prime}$ along the minor arc we have

$$
|M(t)|<e^{(\zeta(3)-1 / 2+\epsilon) N^{2}} .
$$

Using Lemma 3.2 one shows that the integral along the minor arc is relatively small. Then using Lemma 3.1 the integral along the major arc can be approximated using the curve of steepest decent. This gives Wright's asymptotic formula mentioned earlier [15] and illustrates the idea of the circle method.

Recall, we are specifically interested in computing the coefficients of the series $M_{k}(t)=\left.\partial^{k} M_{0}(t, q)\right|_{q=1}$ defined at the start of the section as a means to compute the moments of the random variables $X_{n}^{+}-X_{n}^{-}$. Let us write this series as $M_{k}(t)=$ $F_{k}(t) \cdot M(t)$ then by Wright's two lemmas above we have a good understanding of the singularities of the factor $M(t)$ it remains to analyze $F_{k}(t)$.

Example 3.3. Computing $F_{2}(t)$. Let us differentiate $M_{0}(t, q)$ twice using $\partial=q \frac{d}{d q}$ this gives

$$
\begin{aligned}
& \left(\sum_{m \geq 1} \sum_{k=0}^{m-1} \frac{(1-m+2 k)^{2} q^{4 k-2 m+2} t^{2 m}}{\left(1-q^{2 k+1-m} t^{m}\right)^{2}}+\frac{(1-m+2 k)^{2} q^{2 k-m+1} t^{m}}{\left(1-q^{2 k+1-m} t^{m}\right)}\right) \cdot M_{0}(t, q) \\
& +\left(\sum_{m \geq 1} \sum_{k=0}^{m-1} \frac{(1-m+2 k) q^{2 k-m+1} t^{m}}{1-q^{2 k-m+1} t^{m}}\right)^{2} \cdot M_{0}(t, q) .
\end{aligned}
$$

Then setting $q=1$ we get

$$
\left(\sum_{m \geq 1} \sum_{k=0}^{m-1} \frac{(1+2 k-m)^{2} t^{m}}{\left(1-t^{m}\right)^{2}}\right) \cdot M(t)=\frac{1}{3} \cdot\left(\sum_{m \geq 1} \frac{m\left(m^{2}-1\right) t^{m}}{\left(1-t^{m}\right)^{2}}\right) \cdot M(t)
$$

deducing that

$$
F_{2}(t)=\frac{1}{3} \sum_{m \geq 1} \frac{m\left(m^{2}-1\right) t^{m}}{\left(1-t^{m}\right)^{2}}
$$

In the next two Lemmas we analyze the behavior of $F_{k}(t)$ along the major and minor arcs.

Lemma 3.4. Given $k$ even, there exist constants $N_{0}, K$ such that for all $N>N_{0}$ and $t=e^{z} \in C_{N}^{\prime}$ along the major arc we have

$$
\left|F_{k}(t)-(k-1) ! !(2 \zeta(3))^{k / 2} z^{-2 k}\right|<K N^{2 k-2}
$$

Proof. First let us consider the case $k=2$ used to compute the variance. By what we saw in Example 3.3 above

$$
F_{2}(t)=\frac{1}{3} \sum_{m \geq 1} \frac{m\left(m^{2}-1\right) e^{m z}}{\left(1-e^{m z}\right)^{2}}
$$


By using the Mellin transform $e^{-\tau}=\frac{1}{2 \pi i} \int_{\sigma-i \infty}^{\sigma+i \infty} \Gamma(s) \tau^{-s} d s$ we can express this function as an integral,

$$
\begin{aligned}
F_{2}(t) & =\frac{1}{3} \sum_{m \geq 1} \frac{m\left(m^{2}-1\right) e^{m z}}{\left(1-e^{m z}\right)^{2}}=\frac{1}{3} \sum_{m \geq 1} \sum_{i \geq 1} m\left(m^{2}-1\right) i e^{i m z} \\
& =\frac{1}{6 i \pi} \int_{\sigma-i \infty}^{\sigma+i \infty} \Gamma(s) \sum_{m \geq 1} \sum_{i \geq 1} \frac{m\left(m^{2}-1\right) i}{(i m z)^{s}} d s \\
& =\frac{1}{6 i \pi} \int_{\sigma-i \infty}^{\sigma+i \infty} \Gamma(s) z^{-s} \zeta(s-1)(\zeta(s-3)-\zeta(s-1)) d s .
\end{aligned}
$$

This integrand has a double pole at $s=2$ and a simple pole at $s=4$ coming from the Riemann zeta function while the gamma function contributes simple poles at $s=$ $0,-1,-2, \ldots$. Doing the residue calculus we see that

$$
F_{2}(t)=\frac{\Gamma(4) \zeta(3)}{3} z^{-4}-\frac{2 \gamma \Gamma(2) \zeta(-1)}{3} z^{-2}+O(1)
$$

where $\gamma$ is Euler's constant coming from the Laurent expansion of the zeta function about $s=1$.

Computing the higher order derivatives is essentially an application of Wick's theorem. Indeed if $k=2 r$ then we have

$$
F_{k=2 r}(t)=(k-1) ! !\left(F_{2}(t)\right)^{r}+G_{k}(t)
$$

where the combinatorial coefficient $(k-1)$ !! comes from all possible ways of pairing the $2 r$ differentials $\partial^{2 r}$. Using the product rule for differentiation we see the first term appearing. The remaining terms in $G_{k}(t)$ come from the other terms generated in the product rule. Computing their Mellin transform shows the poles they contribute are of order at least two less.

Lemma 3.5. Given $k$ even, there exists positive constants $N_{0}, C_{k}, A_{k}$ such that for all $N>N_{0}$ and $t=e^{z} \in C_{N}^{\prime \prime}$ along the minor arc we have

$$
\left|F_{k}(t)\right|<C_{k} N^{A_{k}}
$$

Proof. Looking at the definition of $F_{k}(t)$ we see that ultimately it can be written as a finite sums and products of series like

$$
\sum_{n \geq 1} \frac{n^{a} t^{n c}}{\left(1-t^{n}\right)^{b}}
$$

where $a, b, c \in \mathbb{N}$. Each of these sums can be bounded like

$$
\left|\sum_{n \geq 1} \frac{n^{a} t^{n c}}{\left(1-t^{n}\right)^{b}}\right| \leq \sum_{n \geq 1} \frac{n^{a}|t|^{n}}{\left(1-|t|^{n}\right)^{b}}
$$

using the Mellin transform as in the previous lemma gives bounds on this sum like $C_{a, b} N^{A_{a, b}}$ where $|t|=e^{-1 / N}$ and $A_{a, b}, C_{a, b}$ are constants depending only on $a, b$. In total this gives the polynomial bounds claimed. 
Now we have got bounds on the series $M_{k}(t)$ along the major and minor arcs we are ready to estimate the Cauchy integral. Let us define the two quantities we are interested in computing

$$
\begin{aligned}
& I_{k, n}^{\prime}=\frac{1}{2 \pi i} \int_{C_{N}^{\prime}} t^{-n-1} M_{k}(t) d t, \\
& I_{k, n}^{\prime \prime}=\frac{1}{2 \pi i} \int_{C_{N}^{\prime \prime}} t^{-n-1} M_{k}(t) d t .
\end{aligned}
$$

From now onwards we choose $N=(n / 2 \zeta(3))^{1 / 3}$ so that $n=2 \zeta(3) N^{3}$. Then we can get a bound on the integral along the minor arc that is sufficient to show this integral is negligible:

Lemma 3.6. Given $k$ even then for all $\epsilon>0$ there exists positive constants $N_{0}, K, A$ such that for all $N>N_{0}$ we have

$$
\left|I_{k, n}^{\prime \prime}\right|<K \cdot N^{A} \cdot e^{\left(-\frac{1}{2}+\epsilon\right) N^{2}} \cdot e^{3 \zeta(3) N^{2}} .
$$

Proof. Using Lemmas 3.2 and 3.5 we get

$$
\begin{aligned}
\left|I_{k, n}^{\prime \prime}\right| & =\left|\frac{1}{2 \pi i} \int_{C_{N}^{\prime \prime}} F_{k}(t) \cdot M(t) \cdot t^{-n-1} d t\right| \\
& \leq \frac{2 \pi}{|2 \pi i|} \cdot \sup _{C_{N}^{\prime \prime}}\left(\left|F_{k}(t)\right|\right) \cdot \sup _{C_{N}^{\prime \prime}}(|M(t)|) \cdot \sup _{C_{N}^{\prime \prime}}\left(\left|t^{-n-1}\right|\right) \\
& \leq K \cdot N^{A} \cdot e^{(\zeta(3)-1 / 2+\epsilon) N^{2}} \cdot e^{(-n-1) N^{-1}}
\end{aligned}
$$

substituting $n=2 \zeta(3) N^{3}$ gives the result.

From now all that remains is to estimate the integral $I_{n, k}^{\prime}$. Combining Lemmas 3.1 and 3.4 we have

$$
\begin{aligned}
I_{n, k}^{\prime}= & \frac{1}{2 \pi i} \int_{C_{N}^{\prime}} F_{k}(t) M(t) t^{-n-1} d t \\
= & \frac{e^{\zeta^{\prime}(-1)} \cdot(k-1) ! ! \cdot(2 \zeta(3))^{k / 2}}{2 \pi i} \int_{(1-i) / N}^{(1+i) / N} z^{-2 k+1 / 12} e^{\frac{\zeta(3)}{z^{2}+2 \zeta(3) N^{3} z}} d z \\
& +\mathcal{O}\left(N^{-1+2 k-1 / 12-2} e^{\left.3 \zeta(3) N^{2}\right)}\right. \\
= & \frac{n^{2 k / 3}(k-1) ! !}{(2 \zeta(3))^{k / 6}} \cdot \frac{e^{\zeta^{\prime}(-1)} N^{-1 / 12-1}}{2 \pi i} \int_{1-i}^{1+i} v^{-2 k+1 / 12} e^{\zeta(3) N^{2}\left(2 v+v^{-2}\right)} d v \\
& +\mathcal{O}\left(N^{-1+2 k-1 / 12-2} e^{\left.3 \zeta(3) N^{2}\right)}\right.
\end{aligned}
$$

where we set $v=N z$ in the third line.

Notice the prefactor in the above expression is essentially the term we are looking for. From now on we work with the integral

$$
P_{n, k}^{\prime}:=\frac{1}{2 \pi i} \int_{1-i}^{1+i} v^{-2 k+1 / 12} e^{\zeta(3) N^{2}\left(2 v+v^{-2}\right)} d v
$$




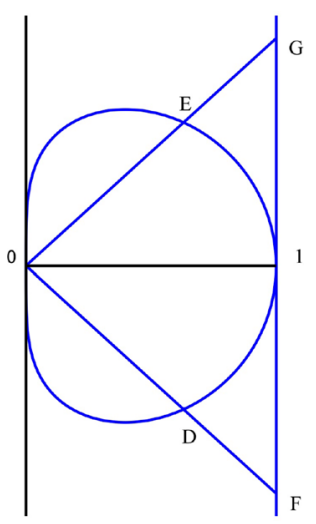

Fig. 3. Curve of steepest descent

basically it will be enough to show that, for large $N$, this is independent of $k$. Then in the limit we have $I_{n, k}^{\prime}=\frac{n^{2 k / 3}(k-1) ! !}{(2 \zeta(3))^{k / 6}} \cdot I_{n, 0}^{\prime}$. We are able to achieve this by localizing the integral $P_{n, k}^{\prime}$ to an even smaller arc using the method of steepest descents.

In the exponent of the integrand we have the function $2 v+v^{-2}$. Roughly speaking the integrand will be largest when this exponent is real. The curve of steepest descent is defined to be the real curve given by $\operatorname{im}\left(2 v+v^{-2}\right)=0$, specifically taking $v=X+i Y$ we have

$$
\left(X^{2}+Y^{2}\right)^{2}=X
$$

This is the closed curve $\mathcal{C}$ meeting the lines $X=0$ at $(0,0), X=1$ at $(1,0), X=-Y$ at $D=\left(2^{-\frac{2}{3}},-2^{-\frac{2}{3}}\right)$, and $X=Y$ at $E=\left(2^{-\frac{2}{3}}, 2^{-\frac{2}{3}}\right)$ as seen in Fig. 3. As in [15] we consider an alternative integral along this curve $\mathcal{C}$ rather than along the straight line $F G$.

Making a branch cut from 0 to $-\infty$ along the real axis we consider the value of $v^{1 / 12}$ which is real and positive at $v=1$ and take the contour for $\mathcal{C}$ parameterized in the anti-clockwise direction. Following Wright we define

$$
\xi_{k}(v)=\frac{v^{-2 k+1 / 12}}{2 \pi i} e^{\zeta(3) N^{2}\left(2 v+v^{-2}\right)} \text { and } J_{k, n}^{\prime}=\int_{\mathcal{C}} \xi_{k}(v) d v .
$$

On the straight lines $E G, D F$ and along the arcs $O E$ and $O D$ we have good bounds on $\xi_{k}(v)$. Since here $\operatorname{Re}\left(v^{-2}\right)=\left(X^{2}-Y^{2}\right) /\left(X^{2}+Y^{2}\right)^{2} \leq 0$ setting $Y=r X$ gives

$$
\left|v^{-2 k+1 / 12} e^{\zeta(3) N^{2}\left(2 v+v^{-2}\right)}\right| \leq X^{-2 k}\left(1+r^{2}\right)^{-k} e^{\zeta(3) N^{2}\left(\frac{1-r^{2}}{\left(1+r^{2}\right)^{2}} X^{-2}\right)} e^{2 \zeta(3) N^{2} X} .
$$

When $r>1$ this tends to zero as $X \rightarrow 0$, and when $r=1$ along the lines $E G, D F$ there are easy bounds. In summary, we have a bound $\left|\xi_{k}(t)\right|<K e^{2 \zeta(3) N^{2} X}$ along these contours $O E, O D, E C$, and $D F$. Using contour integration to compare the original integral $P_{n, k}^{\prime}$ to the new integral $J_{n, k}^{\prime}$ along the curve $\mathcal{C}$ we see that

$$
\left|P_{n, k}^{\prime}-J_{n, k}^{\prime}\right|<\left|\int_{F}^{D} \xi_{k}(v) d v\right|+\left|\int_{D}^{0}\right|+\left|\int_{0}^{E}\right|+\left|\int_{E}^{G}\right|<K e^{2 \zeta(3) N^{2}}
$$


this allows us to integrate along the curve of steepest descent instead. To parameterize this curve we choose

$$
t=-i\left(\frac{v-1}{v}\right)(2 v+1)^{\frac{1}{2}}
$$

so that $t^{2}=3-2 v-v^{-2}$. Now the problem transforms to an integral over the real line

$$
J_{n, k}^{\prime}=e^{3 \zeta(3) N^{2}} \int_{-\infty}^{\infty} \chi_{k}(t) e^{-\zeta(3) N^{2} t^{2}} d t, \quad \text { with } \chi_{k}(t)=\frac{v^{-2 k+1 / 12}}{2 \pi i} \frac{d v}{d t}
$$

The most serious piece of this integral is located about $t=0$ i.e. $v=1$. To understand the behavior here we take a convergent power series $\chi_{k}(t)=\sum_{m=0}^{\infty} a_{m} t^{m}$ in a small neighborhood of $t=0$. Next observe that for some constant $K$ we have

$$
\frac{d v}{d t}=\frac{i v^{2}(2 v+1)^{\frac{1}{2}}}{1+v+v^{2}} \Rightarrow\left|\frac{v^{-2 k+1 / 12}}{2 \pi i} \frac{d v}{d t}\right|<K\left|v^{-2 k}\right|
$$

on all of the real line. Moreover for some possibly larger $K$ we have

$$
\left|v^{-2 k}\right|=\left|3-2 v-t^{2}\right|^{k} \leq K t^{2 k}
$$

on the compliment of the above radius of convergence about $t=0$. All in all we get that

$$
\left|\chi_{k}(t)-\sum_{m=0}^{2 k+1} a_{m} t^{m}\right|<K t^{2 k+2}
$$

over the whole real line. Finally our integral is approximated by

$$
J_{n, k}^{\prime}=\sum_{m=0}^{2 k+1} a_{m} \int_{-\infty}^{\infty} t^{m} e^{-\zeta(3) N^{2} t^{2}} d t+M_{k}
$$

where

$$
\left|M_{k}\right| \leq K \int_{-\infty}^{\infty} t^{2 k+2} e^{-\zeta(3) N^{2} t^{2}} d t<\frac{K}{N^{2 k+3}}
$$

By symmetry all the above odd integrals are zero. The even ones are given by

$$
\int_{-\infty}^{\infty} t^{2 m} e^{\zeta(3) N^{2} t^{2}} d t=\frac{\Gamma\left(m+\frac{1}{2}\right)}{\left(\zeta(3) N^{2}\right)^{m+\frac{1}{2}}}
$$

So to get the leading order asymptotics we need only the constant term $a_{0}=\frac{1}{2 \pi \sqrt{3}}$ in the expansion of $\chi_{k}(t)$. In particular to leading order there is no dependence on $k$ as we claimed earlier. Substituting $N=(n / 2 \zeta(3))^{1 / 3}$ gives the formula described at the end of Section 2. 


\section{Final Remarks}

Some remarks about the asymptotics of DT invariants coming from this investigation. Asymmetry. The $t^{n}$ coefficient of the series $M_{0}\left(t, q^{1 / 2}\right)$ can be seen as giving the contribution to the refined invariants over to the (projective) punctual Hilbert scheme. Here the $q \rightarrow q^{-1}$ symmetry can be interpreted geometrically via the hard Lefschetz theorem for the associated vanishing cycles [4]. Since the refined DT invariants of $\mathbb{C}^{3}$ are given by $M_{3}\left(t, q^{1 / 2}\right)$ their distribution is shifted by the trace of the plane partition coming from $3 X_{n}^{0}$. This shifting is important in defining the refined topological vertex in physics $[8,11]$.

Dimension of moduli space. In his lecture notes on quiver moduli, M. Reineke describes a conjecture on M. Douglas on the asymptotic growth of Euler numbers of spaces of representations of Kronecker quivers [13]. Reineke proposes a generalization of the conjecture would give

$$
\ln \left(\chi\left(M_{d}(Q)\right)\right) \sim C_{Q} \sqrt{\operatorname{dim}\left(M_{d}(Q)\right)}
$$

where $\left.M_{d}(Q)\right)$ is a suitable smooth model for the quiver moduli, $d$ is a large dimension vector, and $C_{Q}$ is an interesting constant to be determined.

While writing this paper we noticed a similar behavior for asymptotic growth in the case of the Hilbert scheme of points. For $k \geq 2$ it appears that,

$$
\ln \left(\chi\left(\operatorname{Hilb}^{n}\left(\mathbb{C}^{k}\right)\right)\right) \sim C_{k} \sqrt{\operatorname{dim}\left(\operatorname{Hilb}^{n}\left(\mathbb{C}^{k}\right)\right)}
$$

for $n$ very large. The asymptotic behavior of $\operatorname{dim}\left(\operatorname{Hilb}^{n}\left(\mathbb{C}^{k}\right)\right)$ was determined in [3]. For $k=2,3$ the growth of $\chi\left(\operatorname{Hilb}^{n}\left(\mathbb{C}^{k}\right)\right)$ is know by [7] and [15] respectively. The constant $C_{2}$ is determined but we can only get bounds on the constant $C_{3}$.

Acknowledgements. Primarily, I wish to thank T. Hausel for his hospitality in inviting me to EPFL and sharing the ideas that lead to this paper. Also thanks to J. Bryan, R. Pandharipande, and B. Young for their support and comments, and to M. Marcolli who was my mentor at MSRI where this paper was written.

This paper was finished during a postdoc at MSRI in the spring 2013 program Non-Commutative Algebraic Geometry and Representation Theory. Normally I am a postdoc at ETH Zürich in the research group of R. Pandharipande sponsored by Swiss grant 200021143274.

\section{References}

1. Andrews, G.E.: The Theory of Partitions. Advanced Book Program. Addison-Wesley Pub. Co., Boston (1976)

2. Behrend, K., Bryan, J., Szendrői, B.: Degree zero motivic Donaldson-Thomas invariants. Invent. Math. 192, 111-160 (2013)

3. Briancon, J., Iarrobino, A.: Dimension of the punctual Hilbert scheme. J. Algebra 55, 536-544 (1978)

4. Davison, B., Maulik, D., Schuermann, J., Szendroi, B.: Purity for graded potentials and quantum cluster positivity. arXiv:1307.3379

5. Freed, D.S.: Five Lectures on Supersymmetry. American Mathematical Society, Providence (1999)

6. Harvey, J.A., Moore, G.: On the algebras of BPS states. Commun. Math. Phys. 197(3), 489-519 (1998)

7. Hardy, G.H., Ramanujan, S.: Asymptotic formulae in combinatory analysis. Proc. Lond. Math. Soc. 17, 75 115 (1918)

8. Iqbal, A., Kozaz, C., Vafa, C.: The refined topological vertex. J. High Energy Phys. 10, 069 (2009)

9. Kamenov, E.P., Mutafchiev, L.R.: The limiting distribution of the trace of a random plane partition. Acta Math. Hung. 117, 293-314 (2007) 
10. Kontsevich, M., Soibelman, Y.: Cohomological Hall algebra, exponential Hodge structures and motivic Donaldson-Thomas invariants. Commun. Number Theory Phys. 5, 231-252 (2011)

11. Morrison, A., Mozgovoy, S., Nagao, K., Szendrői, B.: Motivic Donaldson-Thomas invariants of the conifold and the refined topological vertex. Adv. Math. 230, 2065-2093 (2012)

12. Okounkov, A., Reshetikhin, N.: Random skew plane partitions and the Pearcey process. Commun. Math. Phys. 269(3), 571-609 (2007)

13. Reineke, M.: Moduli of representations of quivers. In: Proceedings of the ICRA XII Conference, Torun (2007)

14. Reineke, M.: Cohomology of non-commutative Hilbert schemes. Algebras Represent. Theory 8, 541-561 (2005)

15. Wright, E.M.: Asymptotic partition formulae I. Plane partitions. Q. J. Math. 2(1), 177189 (1931)

Communicated by N. Reshetikhin 